\title{
EXPERIENCIAS
}

\section{UN INSTRUMENTO PARA EL ANALISIS DE LOS LIBROS DE TEXTO DESDE UNA ORIENTACIÓN SOCIOLABORAL NO SESGADA}

\author{
A TOOL TO ANALYZE TEXT BOOKS FOR AN UNBIASED \\ OCCUPATIONAL GUIDANCE
}

Cristina Miranda Santana*

Univesidad de Las Palmas de Gran Canaria

\begin{abstract}
RESUMEN
El valor de este trabajo se enmarca desde lo que podemos denominar diagnóstico en educación y se fundamenta en aportar un instrumento que nos permite analizar en qué medida los materiales didácticos utilizados habitualmente en el desarrollo del curriculum escolar atienden a una educación sociolaboral no sesgada. En concreto los libros de texto al ser los recursos más utilizados por el profesorado. Asimismo, aportamos las rejillas de análisis de los códigos lingüísticos e icónicos.

Palabras claves: Desarrollo de la Carrera, orientación y educación sociolaboral, libros de texto, género, matrices, diagnóstico en educación, curriculum.
\end{abstract}

\begin{abstract}
The importance of this report its based in what we can call educational diagnosis and it provides an instrument that will allow us to analyse in what measure the didactic materials used in the curriculum development confirm non-stereotyped career education specifically the text books because they are the most widely used instruments amongst teachers. We also provide a matrix with the linguistic and image codes.
\end{abstract}

Palabras claves: Career Development, Career guidance and Career education, text book, gender, matrix, educational diagnosis, curriculum.

* Doctora en Psicopedagogía. Profesora Asociada de la Universidad de Las Plamas de Gran Canaria, en la que imparte docencia en: Orientación Profesional y Ocupacional, Tutoría y orientación y Diagnóstico en Orientación. Directora de Información al estudiante en la Universidad de Las Palmas de Gran Camaria. Líneas de investigación: orientación y educación sociolaboral no sesgada, transición a la vida adulta y activa y orientación en colectivos de difícil inserción sociolaboral. 


\section{Introducción}

La diversidad en la etapa de Educación Secundaria Obligatoria es un hecho real. En nuestro contexto, en apenas casi 50 años, la escuela pasa de estar ocupada casi en exclusividad, por un grupo homogéneo de estudiantes, a encontrarnos en ella con mujeres y con alumnos(as) pertenecientes a otras razas y a otras culturas, con algún tipo de discapacidad y de diferente condición económica, como fruto de la escolarización obligatoria. El Libro Blanco de la Reforma del Sistema Educativo (1989) asume que la finalidad de lo comprensivo debe fundamentarse en:

i) facilitar una formación polivalente al alumnado de un mismo centro y de un mismo curriculum básico,

ii) retardar su separación en ramas diferentes de formación, y

iii) reducir las diferencias educativas por sexo.

Sin embargo, los conocimientos que se pretenden enseñar no son neutros $y$, tampoco atienden a la especificidad de nuestro alumnado-, en ninguno de los términos ya expuestos Feito (1997); Así, la posibilidad de transmitir conocimientos que no estén vinculados al entorno del alumnado es una tarea sin sentido.

En estos momentos, los libros de texto se configuran en el actual sistema educativo como curriculas, prescindiendo en cierta medida del curriculum oficial (Area, 1991). Si somos conscientes de estas dos realidades, la diversidad de nuestro alumnado y la utilización de los libros de texto como fuente de conocimiento para los alumnos y las alumnas y también, como fuente estructurada de enseñanza para el profesorado (Area, 1991:68). Podemos reconocer que el análisis de los libros de texto nos permite identificar hasta qué punto el profesorado incorpora en sus propuestas curriculares la interacción del género respecto al proyecto sociolaboral de los(as) jóvenes. Partiendo de estos supuestos, un grupo de profesores y profesoras de $3^{\circ}$ de la ESO que desarrolla su labor en un IES de la Comunidad Autónoma de Canaria con la ayuda de una asesora externa se propone reconocer en qué medida los libros de texto utilizados como soporte en el desarrollo de las materias incorpora una educación sociolaboral de base no sesgada.

Cuando nos acercamos a consultar otras experiencias que tienen como finalidad el análisis de medios didácticos podemos comprobar que la mayoría de los estudios realizados sobre la representatividad de propuestas para la atención al género en los materiales curriculares se centra en el análisis de los libros de texto, ya que se articulan como las propuestas curriculares preelaboradas más utilizadas por la mayoría del profesorado.

En cuanto al contenido del análisis -atención al género y a la dimensión sociolaboral-, observamos una mayor tradición en el contexto anglosajón que en el español en la dimensión género, no así en lo referente a la dimensión sociolaboral, pues no se da -sorprendentemente para nosotros(as) - en ninguno de los dos contextos.

En España este tipo de análisis comienza a tomar relevancia en la década de los ochenta y a producir información sistematizada en los noventa. Los(as) distintos(as) autores(as) se han centrado en unas ocasiones en el análisis del código lingüístico exclusivamente (Blom, Waite \& Zimet, 1970; Frasher \& Walker, 1972; Saario, Jackelin \& Tittle, 1973; Potter, 1977). Otros(as) han estudiado las ilustraciones (Weitzman \& Rizzo, 1974; Powell \& García, 1985). Por último, también se ha trabajado ambos aspectos de los libros de texto 
(1971; DeCrow, 1972; Graebner, 1972; Marten \& Martlin 1976; Kepner \& Kochn, 1977; and Rupley, García \& Longnion, 1981).

En el contexto español existen aproximaciones formalizadas referidas al análisis del material curricular desde una óptica de género, algunas caracterizadas por compilar en el instrumento de análisis aspectos referidos al texto y a la imagen (Garreta y Careaga, 1987; Marco, 1992; Zaitegi y Urruzola, 1993; Espín, Moreno et al, 1996; Álvarez y Miranda, 1997).

Desde una perspectiva coeducativa existen múltiples instrumentos para el análisis de los libros de texto, aunque no muchos que nos permiten realizar un análisis de estereotipos sociolaborales y sesgos sexistas (Eklund, 1988; Eichler, 1991; Potter, Rosser, 1992; Zaitegi y Urruzola, 1993; Molina y Zurriarraín, 1994; Romero, 1994; Madonar, 1994; Ferrer, 1994; Berroeta, 1994; Guerrero, 1994; Alcalá, 1994; Espín et al, 1996).

\section{Un instrumento para el análisis del código icónico y lingüístico en la interacción del género respecto al proyecto sociolaboral de los(as) jovenes}

Afrontar este reto supuso generar un instrumento que permita al profesorado analizar sus libros de texto. Con este fin revisamos algunos de los instrumentos que se han centrado en el análisis de los libros de texto, e inspirándonos en ellos generamos matrices de análisis,

i) matriz de análisis del código icónico para los libros de texto,

ii) matriz de análisis del código lingüístico para los libros de texto.

A continuación presentamos las matrices de análisis y definimos el conjunto de categorías que establecimos para el desarrollo del análisis. 


\section{Matriz para el análisis del código icónico en los libros de texto en la interacción} del género en el proyecto sociolaboral de los(as) jóvenes

\section{Cuadro 1: Matriz de análisis del código icónico para los libros de texto.}

\begin{tabular}{|c|c|c|c|c|c|c|c|c|c|c|}
\hline \multicolumn{4}{|c|}{ MATERIA: } & \multicolumn{4}{|c|}{ LIBRO: } & \multicolumn{3}{|c|}{ UNIDAD: } \\
\hline $\begin{array}{l}\text { ANALLISI } \\
\text { IMAGEN }\end{array}$ & \multicolumn{3}{|c|}{ PERSONAS: } & & & & \multicolumn{4}{|c|}{ SEXO FEMENINO: } \\
\hline \multirow[t]{2}{*}{$N^{\circ}$ DIBUJ } & \multicolumn{4}{|c|}{ TIPO DE DIBUJO } & \multicolumn{3}{|c|}{ No DE PERSON. } & \multicolumn{3}{|c|}{ PROTAGONISMO } \\
\hline & $\begin{array}{l}\frac{1}{p} \\
\text { के } \\
\text { Dे }\end{array}$ & \multicolumn{2}{|c|}{ 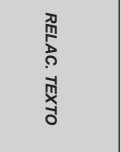 } & 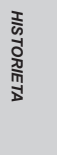 & 3 & \multicolumn{2}{|c|}{$\pi$} & \multicolumn{2}{|r|}{ 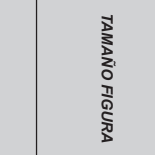 } & 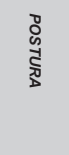 \\
\hline \multicolumn{11}{|c|}{ ROLES PROFESIONALES } \\
\hline \multirow[t]{2}{*}{ ACTIVIDA } & \multicolumn{2}{|c|}{ SECTOR } & \multicolumn{2}{|c|}{ SEXO } & \multicolumn{2}{|c|}{ TIPO DE TRABAJO } & \multicolumn{2}{|c|}{ TIPO ESCENARIO } & \multicolumn{2}{|c|}{ DIVISIÓN TRABAJO } \\
\hline & 疋 & 要 & $\begin{array}{l}0 \\
\frac{1}{0} \\
\frac{0}{0} \\
\frac{0}{0} \\
\frac{1}{2}\end{array}$ & $\begin{array}{l}\frac{m}{\sum_{0}} \\
\frac{m}{0} \\
0\end{array}$ & 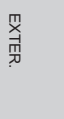 & $\begin{array}{l}\text { 总 } \\
\text { 罗 }\end{array}$ & 竞 & $\sum_{\text {t. }}^{3}$ & 产 & 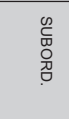 \\
\hline \multirow[t]{3}{*}{$N^{\circ} D I B L$} & \multicolumn{3}{|c|}{ ESTEREOTIPO ROL SOCIAL } & \multicolumn{4}{|c|}{ ROL POLITICO } & \multicolumn{3}{|c|}{ ROL FAMILIAR } \\
\hline & \multicolumn{2}{|c|}{ 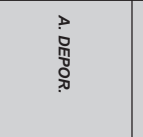 } & 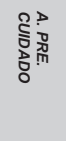 & \multicolumn{2}{|c|}{ 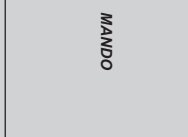 } & \multicolumn{2}{|c|}{$\begin{array}{l}\text { 量 } \\
\text { î. } \\
\text { i. }\end{array}$} & \multicolumn{2}{|c|}{ 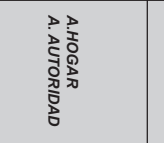 } & \\
\hline & & & & 斀 & $\begin{array}{l}\frac{8}{0} \\
\frac{0}{5} \\
5\end{array}$ & 署 & 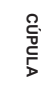 & & & \\
\hline \multicolumn{11}{|c|}{ ROLES PROFESIONALES } \\
\hline \multicolumn{2}{|c|}{ NIVEL DE RESPONSABIILIDAD } & \multicolumn{3}{|c|}{ CONTRIBUCIÓN } & \multicolumn{3}{|c|}{ RELACIÓN LABORAL } & \multicolumn{3}{|c|}{ TIPO DE EMPRESA } \\
\hline : & $£$ & & 2 & \% & & & 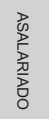 & & & 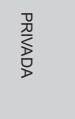 \\
\hline & & & & & & & & & & \\
\hline
\end{tabular}

1. Tipo de dibujo: alude a la funcionalidad del dibujo respecto al discurso.

1.1. Dibujos aislados: son aquellos que hacen referencia a la unidad del libro en el que se encuentra; carecen de valor en el discurso y actuarán como motivador. Podemos afirmar que es una ilustración sin más.

1.2. Dibujos relacionados por la idea de un texto: hacen una referencia explicativa al texto; sin él, no se podría entender el aspecto que se quiere comunicar.

1.3. Historietas: son aquellos dibujos que hacen alusión a un relato ilustrado en el que pueden reflejarse una o múltiples persona. 
A partir de cada imagen e independientemente del tipo que sea va a ser analizada en función de los siguientes aspectos:

2. $N^{o}$ de personas: hace referencia a la cantidad de hombres y mujeres que aparecen en cada ilustración. El objeto está en conocer la predominancia o no de los géneros.

3. Protagonismo: qué personajes, ya sean masculinos o femeninos, representan la acción. El objeto es conocer si existe preponderancia de un género sobre otro. Para señalar los aspectos que definen este protagonismo hemos utilizado criterios de la comunicación visual tales como el plano, el tamaño de la figura o la postura:

3.1. El Plano en el que aparece la figura: hace referencia a la representatividad del personaje. Un segundo plano indica un menor protagonismo respecto a un primer plano.

3.2. El tamaño de la figura, muchas veces, se utiliza para aumentar a un personaje o un hecho frente a otros y así darle mayor relevancia a esa persona o acción.

3.3. La postura, relacionada con el lenguaje corporal, a partir de indicadores de protección, de violencia, de quietud, de actividad, de comprensión, etc. Así podremos corroborar si se da o no actividad y qué tipo de actividad es propia del género femenino y masculino.

4. Estereotipo de rol de género: el estereotipo de género es una derivación del estereotipo de rol social. Tajfel (1981) define estereotipo social como las imágenes mentales simplificadas de personas o grupos que comparten un número considerable de personas. Existe, entre ellas, consenso social respecto a esta situación. De aquí deducimos que el estereotipo de género es aquella -o aquellas- imagen mental referida a la posición que un colectivo o persona ocupa en la estructura social por ser mujer u hombre.

5. Estereotipo de personalidad: hace referencia a las características de personalidad con las que normalmente se asocia al género masculino (violencia, competitividad, actividad, protección, inteligencia, creatividad, etc.) y al femenino (ternura, delicadeza, solidaridad, diálogo, sumisión, falta de iniciativa). Buscábamos conocer si se transmitían estos estereotipos y cuáles eran.

6. Estereotipos de rol social: alude a las funciones sociales que son atribuidas a hombres y mujeres en función de su sexo. Generalmente, a las mujeres le son atribuidas funciones referidas al cuidado y a la crianza de la descendencia, mientras que a los hombres le son asignadas funciones relacionadas con la contribución económica para el mantenimiento de esa descendencia.

7. Estereotipo de rol político: atiende al cometido político asignado a unas y a otros en función de su sexo. Mientras que a la mujer se le asignan funciones de administración de recursos, a los hombres le son establecidas actuaciones de gestión y decisión sobre los recursos.

8. Estereotipo de rol familiar: referido a la asignación de funciones dadas a hombres y mujeres en el ámbito de lo privado, en el hogar. Así, ellos aparecen con una imagen de autoridad mientras que ellas siempre están asociadas a actividades de mantenimiento del hogar. 
9. Estereotipo de rol profesional: hace referencia a la distribución de actividades profesionales en función del género. Por ello, se entiende que los chicos desarrollan un mejor desempeño de profesiones científico-tecnológicas y las chicas en profesiones de carácter socio-lingüístico-humanístico.

9.1. Sector: hacemos referencia al sector productivo-económico en el que se integra la actividad (primario, secundario y terciario)

9.2. Actividad: nos referimos a la definición de la tarea que desempeña.

9.3. Sexo: aludimos al de género de la persona que está desempeñando esa actividad.

9.4. Tipo de trabajo: consideramos en este aspecto la contribución económica del trabajo desempeñado. Incorporamos dos subcategorías: trabajo remunerado (por el que se recibe una contraprestación económica) y trabajo no remunerado (no existe contraprestación económica, aunque se pueden dar otras contraprestaciones de forma implícita: afectivas, de reconocimiento social, etc.).

9.5. Características de trabajo: hacemos referencia a la dimensión intelectual (aquél en el que el(a) profesional tiene la posibilidad de tomar decisiones y de crear a partir de su criterio. Generalmente no tiene contacto directo con el desarrollo de las propuestas, sino a través de otros(as) profesionales) vs. manual (aquel trabajo en el que apenas toman decisiones y sus actuaciones son prescritas por otras personas de mayor rango) en su realización.

9.6. Nivel de responsabilidad: hace referencia a la competencia en el desempeño profesional.

9.6.1. Mando: cuando la persona está obligada a gestionar recursos ya sean materiales o humanos. En él se consideran distintos niveles y, a mayor amplitud del campo, se da una cota más extensa para una toma de decisiones de forma autónoma.

9.6.2. Subordinado $(a)$ : son tareas o acciones desarrolladas por profesionales bajo la supervisión de otras(os).

9.7. Contribución: alude a cómo la tarea desempeñada por las(os) profesionales va a favorecer la participación en distintas parcelas: económica, política, social.

9.8. Relación laboral: nos referimos a si la actividad desempeñada está sujeta a un contrato en el que se fijan condiciones respecto a horarios, salario, tipo de trabajo, etc. o si, por el contrario, el(a) profesional es su propio empleador(a).

9.9. Tipo de empresa: queremos hacer hincapié en si la relación laboral se establece en un mercado de trabajo público en donde las condiciones de acceso y promoción están perfectamente definidas o si por el contrario, se dan en un contexto privado en donde el empleador puede, respetando la ley vigente, establecer sus propios requisitos para favorecer el desarrollo de su empresa, restringiendo o facilitando el acceso a ella. 
Matriz para el análisis del código lingüístico en los libros de texto en la interacción del género en el proyecto sociolaboral de los(as) jóvenes

Cuadro 2: Matriz de análisis del código lingüí́stico para los libros de texto.

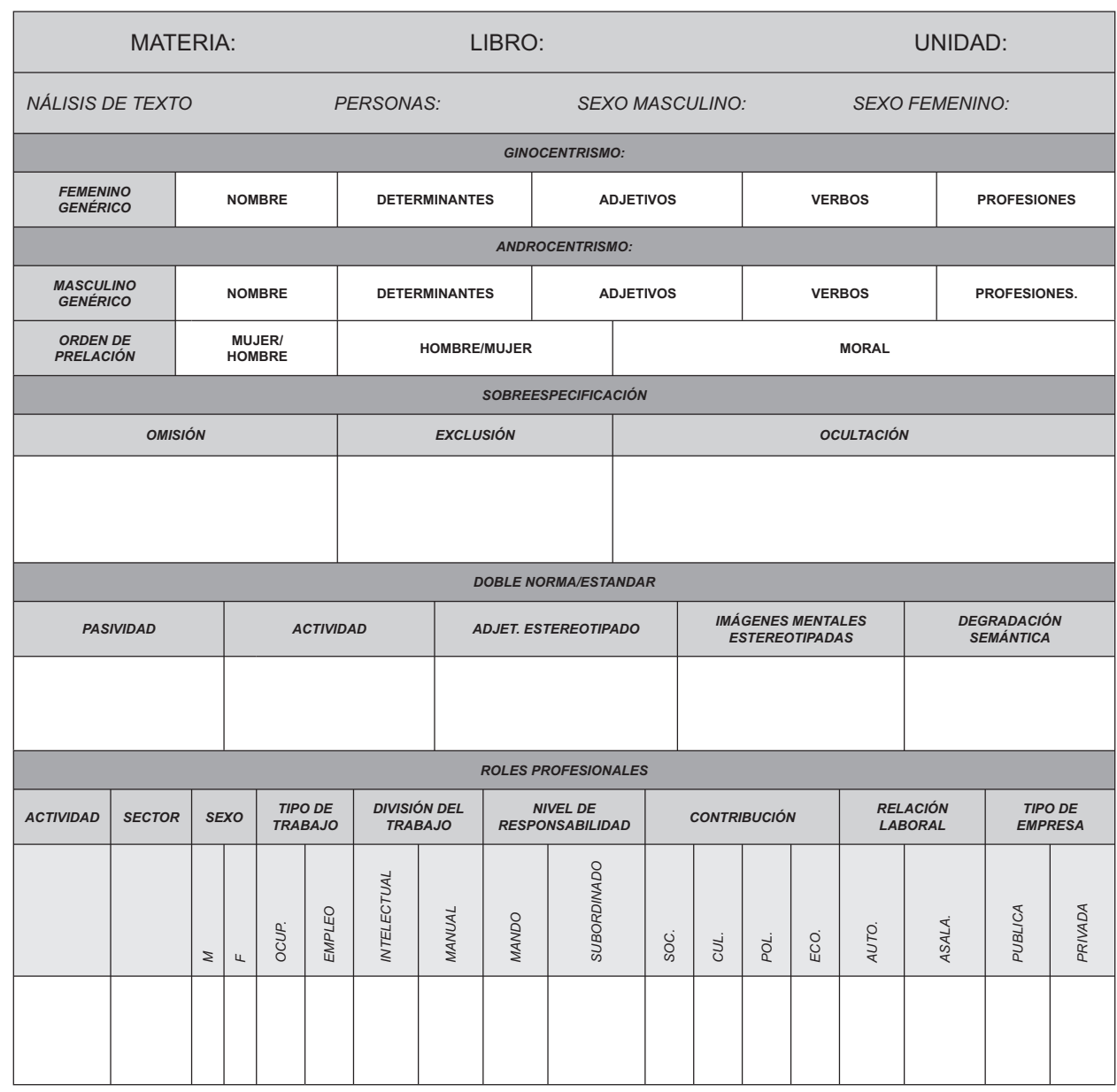

1. Ginocentrismo: hace referencia a la visión femenina que se tiene en la conceptualización-expresión lingüística de los aconteceres cotidianos.

1.1. Femenino genérico: cuando utilizamos el género femenino para referirnos a mujeres y a hombres. Registramos el femenino genérico en los determinantes (utilizar "las jóvenes" por los y las jóvenes), en los adjetivos ("adolescentes idiotas", incorporando a los y las adolescentes idiotas), en los verbos ("salgan todas al recreo", incorporando a todos los alumnos y a todas las alumnas) y en las profesiones ("enfermeras", por enfermeras y enfermeros). 
1.2. Orden de prelación: se refiere al uso constante de un género antes que el otro. Así nos referimos a la madre y al padre (en este caso desconocemos que se dé orden de prelación).

2. Androcentrismo: hace referencia a la visión masculina que se tiene en la conceptualización-expresión lingüística de los aconteceres cotidianos.

2.1. Masculino genérico: cuando utilizamos el género masculino para referirnos a hombres y a mujeres. Registramos el masculino genérico en los nombres ("los abuelos", abuelo y abuela), en los determinantes ("los adolescentes", incorporando a las adolescentes y a los adolescentes), en los adjetivos ("adolescentes desorientados", incorporando a adolescentes desorientadas y desorientados), en los verbos ("saquen todos el libro de texto", refiriéndose a un grupo de chicas) y en las profesiones ("arquitectos" por arquitectas y arquitectos).

2.2. Orden de Prelación: hace alusión a la utilización constante de un género antes que el otro. En la escritura niño(a) o en referencia a la dependencia de orden moral de un género respecto a otro (el marido y su esposa).

3. Sobreespecificación: nos referimos a aquellos conceptos que identifican una situación para un sólo género.

3.1. Omisión: cuando un concepto no es personalizado lleva a que sea atribuido a un solo género (que por defecto es el masculino). Por ejemplo, no es lo mismo decir "el médico no viene hoy" que "D. José, el médico, no viene hoy".

3.2. Exclusión: se establece cuando un concepto es atribuido a un sólo género, por lo que el otro queda excluido (ama de casa).

3.3. Por ocultación: cuando un concepto se refiere a un sólo sexo pudiendo ser aplicable a ambos ("enfermera", "auxiliar"...).

4. Doble Norma/Estándar: se refiere a la estructura gramatical tanto en la expresión escrita como oral en relación el desarrollo de actividades, al establecimiento de juicios distintos acerca de las mismas actuaciones según sean desempeñadas por mujeres u hombres.

4.1. Pasividad/Actividad: un género siempre es nombrado en activo y el otro en pasivo. "Todos en la guerra tenemos nuestras funciones, ellos hacen las batallas y ellas se ocupan de la enfermería y la administración".

4.2. Adjetivos estereotipados: planteamos que unas cualidades son propias de un género cuando los dos pueden ser partícipes (madre proteccionista/padre proteccionista).

4.3. Imágenes mentales estereotipadas: atribuyen a uno u otro sexo visiones sesgadas y socialmente aceptadas. Por ejemplo: Don Juan, Maruja...

4.4. Degradación semántica: cuando el significado de la palabra tiene un sentido peyorativo al cambiar de género. Por ejemplo: hombre público, mujer pública.

5. Roles profesionales: hace referencia a la distribución de actividades profesionales en función del género. Así, se entiende que los chicos desarrollan un mejor desempeño de profesiones científico-tecnológicas y las chicas en profesiones de carácter sociolingüístico. 
5.1. Sector: hacemos referencia al sector productivo-económico en el que se integra la actividad (primario, secundario y terciario).

5.2. Actividad: nos referimos a la definición de la tarea que desempeña.

5.3. Sexo: de qué sexo es la persona que está desempeñando esa actividad.

5.4. Tipo de trabajo: consideramos en este aspecto la contribución económica del trabajo desempeñado. Incorporamos dos subcategorías: trabajo remunerado (por el que se recibe una contraprestación económica) y trabajo no remunerado (no existe contraprestación económica, aunque se pueden dar otras contraprestaciones de forma implícita: afectivas, de reconocimiento social, etc.).

5.5. División del trabajo: Hacemos referencia a la dimensión intelectual vs manual en su realización.

C. Trabajo intelectual: entendemos a aquél en el que el(a) profesional tiene la posibilidad de tomar decisiones y de crear a partir de su criterio. Generalmente no tiene contacto directo con el desarrollo de las propuestas sino a través de otros(as) profesionales.

D. Trabajo manual: son aquellos trabajos en donde la toma de decisiones es a pequeña escala o casi nula y sus actuaciones son prescritas por otras personas con un rango mayor.

5.6. Nivel de responsabilidad: hace referencia a la competencia en el desempeño profesional.

E. Mando: cuando la persona está obligada a gestionar recursos ya sean materiales o humanos. En él se consideran distintos niveles y, a mayor amplitud del campo se da una cota más amplia para la toma de decisiones de forma más autónoma.

F. Subordinado $(a)$ : son tareas o acciones desarrolladas por profesionales baj o la supervisión de otras(os).

5.7. Contribución: alude a cómo la tarea desempeñada por las(os) profesionales va a favorecer/participar de distintas parcelas: económica, política, social.

5.8. Relación laboral: nos referimos a si la actividad desempeñada está sujeta a un contrato en el que se fijan condiciones respecto a horarios, salario, tipo de trabajo, etc. o si, por el contrario, el(a) profesional se configura como su propio(a) empleador(a).

5.9. Tipo de empresa: queremos hacer hincapié en si la relación laboral se establece en un mercado de trabajo público en donde las condiciones de acceso y promoción están perfectamente definidas o si, por el contrario, se dan en un contexto privado en donde el empleador puede, respetando la ley vigente establecer sus propios requisitos para favorecer el desarrollo de su empresa, restringiendo o facilitando el acceso a ella.

En síntesis, podríamos afirmar que el valor de estos instrumentos: las matrices, no esta sólo en sus capacidades para identificar-diagnosticar hasta qué punto los libros de texto de las diferentes materias responde a una educación sociolaboral de base no sesgada; sino también, y por lo que es considerado más importante, por su virtualidad para orientar en la intervención, en la elaboración de materiales didácticos contextualizados que permitan la in- 
teracción del género en los proyectos sociolaborales de los(as) jóvenes. Este es el caso, generar un material didáctico que permita la integración de elementos que favorecen el desarrollo de la madurez vocacional desde una perspectiva no sesgada -disciplinar o interdisciplinar- en el curriculum de las materias.

\section{Referencias bibliográficas}

Alcalá Cortijo, P. (1994). Con faldas y en la Ciencia. Experiencia de orientación no sexista hacia las ciencias. Comunicación presentada en las Jornadas de Orientación Académio-Vocacional para una Toma de Decisión no Discriminatoria. Emakunde/Instituto Vasco de la Mujer. Vitoria-Gasteiz.

Álvarez, P. y Miranda, C. (1997). La transmisión de estereotipos sexistas a traves del libro de texto: su incidencia en la toma de decisiones vocacionales. Comunicación presentada a las VIII Jornadas Nacionales de la Asociación Española para la Orientación Escolar y Profesional: La orientación educativa y la intervención psicopedagógica integradas en el curriculum. Valencia.

Area Moreira, M. (1991). Los Medios, los profesores y el currículo. Barcelona: Sendai ediciones

DeCrow, Roger (1972). National Right to Read Partners.

Eichler, M. (1991). Non sexist research methods. A practical guid. London: Routledge, Chapman and Holl, Inc.

Espín, J. et al. (1996). Análisis de recursos educativos desde la perspectiva no sexista. Barcelona: Laertes.

Espín, J. et al. (1996). "Mujer y orientación: por una orientación para la igualdad de oportunidades". Revista de Orientación Profesional (ROP), 7, 11, 59-75.

Feito, R. (1997). "El contenido de la enseñanza”. En M. Fernández Enguita, Sociología de las instituciones de educación secundaria (pp. 124-133). Barcelona: ICE/Horsori.

Frasher, R. Walker, A. (1972). "Sex Roles in Early Reading Textbooks". Reading Teacher, 25, 8, 74149.

Garreta, N. y Careaga, P. (1987). Modelos masculino y femenino en los libros de texto de EGB. Madrid: Instituto de la Mujer.

Graebner, D. B. (1972). "A decade of sexism in readers". Reading teacher, 26, 1, 52-58.

Guerrero Díaz, A. (1994). Orientación profesional coeducativa. Comunicación presentada en las Jornadas de Orientación Académio-Vocacional para una Toma de Decisión no Discriminatoria. Emakunde/Instituto Vasco de la Mujer. Vitoria-Gasteiz.

Kepner, H. y Koehn, L. (1977). "Sex roles in mathematics: a study of the status of sex stereotypes in elementary mathematics texts arithmetic teacher". Journal of Education, 24, 5, 379-385.

Marco, R. et al. (1992). Modelos informáticos para el asesoramiento vocacional. Ponencia presentada en la European Conference about Information Technology, 2, 39, 185-192.

Marten, L. y Matlin, M. (1976). "Does sexism in elementary readers still exist?". Reading Teacher; 29, 8, 764-67.

Molina Guerrero, M. J. y Zurriarrain Azagra, I. (1994): La adolescencia, momento de elección. Comunicación presentada a las Jornadas de Orientación académio-vocacional para una toma de decisión no discriminatoria. Emakunde/Instituto Vasco de la Mujer. Vitoria-Gasteiz.

Potter, E., y Rosser, S. (1992). "Factors in Life Science Textbooks That May Deter Girls' Interest in Science". Journal of Research in Science Teaching, 29, 7, 669-86.

Potter, G. (1977). "The promise of interdisciplinarity and its problems". Ramapo Papers, 1, 3. 
Powell, R. y García, J. (1985). The portrayal of minorities and women in selected elementary science series.

Romero, L. (1992). “Acerca de la orientación profesional”. Nuestra Escuela, 130, 29-33.

Rupley, García y Lonfnion. (1981). "Sex role portrayal in reading materials: implications for the 1980s". Reading Teacher, 34, 7, 786-91.

Shoemaker, B. y Eklund, J. (1989). "Integrative education: a curriculum for the twenty-first century". OSSC Bulletin, 33, 2.

Weitzman, L. y Rizzo, D. (1974). Biased textbooks: images of males and females in elementary school textbooks in five subject areas; What you can do about biased textbooks.

Zaitegi, N. y Urruzola, J. M. (1993). Cómo elaborar y seleccionar materiales coeducativos. Bilbao: Departamento de Educación, Universidades e Investigación del Gobierno Vasco.

Fecha de recepción: 27-05-02

Fecha de revisión: 19-01-04

Fecha de aceptación: 12-05-04 